REVISTA DE DERECHO UNED, NÚM. 27, 2021

\title{
DE LAS CLASES MAGISTRALES AL MÉTODO DEL CASO ANTE SUPUESTOS SOBRE HERENCIA, NASCITURUS Y POSTUMUS
}

\author{
FROM MASTER CLASSES TO THE CASE METHOD TO CASES \\ ON HERITAGE, NASCITURUS AND POSTUMUS
}

\author{
Elisa Muñoz CataláN \\ Doctora en Derecho, Premio extraordinario de Doctorado \\ Tutora con venia docencia. UNED \\ Doctora en Derecho acreditada \\ Universidad Internacional de La Rioja - España \\ elisa.munoz@unir.net
}

Sumario: I. Introducción; II. Objetivos planteados y contenidos propuestos en el supuesto de doble matrimonio, nasciturus e hijo póstumo ante la herencia; II.A. Justificación; II.B. Objetivos planteados para aplicar el método del caso; II.C. Contenidos conceptuales, procedimentales y actitudinales; III. Desarrollo de la actividad en clase, de forma presencial y virtual; III.A. Primera sesión; III.B. Segunda sesión; iv. Temporalización recomendada; V. Recursos; V.A. Recursos materiales (material "aprender a aprender»); V.B. Recursos ambientales, estructurales y propios del ámbito universitario; VI. Evaluación: criterios e instrumentos; VII. Conclusiones y resultados; VIII. Bibliografía; VIII.A. Referencias bibliográficas; VIII.B. Fuentes jurisprudenciales, legislativas y/o electrónicas.

Resumen: Con la realización del presente trabajo, nuestra intención es reparar en la importancia actual de aplicar el método del caso en la enseñanza online del Derecho Civil, frente a las tradicionales clases teóricas y magistrales que han caracterizado la Carrera de Derecho. Examinando, muy especialmente, el alcance de las exi- 
gencias metodológicas surgidas con los planes del estudio Grado en Derecho en el marco del EEES y con la necesidad de una docencia universitaria online por la pandemia mundial que estamos sufriendo desde comienzos de 2020 a causa del coronavirus. Por tanto, con la puesta en práctica de este supuesto que versa sobre doble matrimonio, nasciturus e hijo póstumo y herencia desde Roma hasta nuestros días, intentamos acercar al alumnado al casuismo jurisprudencial aplicado al Derecho Civil español; de este modo, comprenderán que lo importante no es dar la solución al supuesto planteado, sino razonar jurídicamente consiguiendo, a través de debates online y en grupos, un conocimiento más profundo de las reglas e instituciones aplicables.

Palabras clave: método del caso, hijo póstumo, nasciturus, herencia, Grado en Derecho.

Abstract: With the realization of this work, our intention is to repair the current importance of applying the case method in the online teaching of Civil Law, compared to the traditional theoretical and master classes that have characterized Law Studies. Examining, especially, in the reach of the methodological requirements emerged with the plans of study Law Degree under the EEES and with the need for online university teaching due to the global pandemic that we have been suffering since the beginning of 2020 due to the coronavirus. Therefore, with the implementation of this case, that is about double marriage, nasciturus and posthumous son and heritage from Rome to the present, we try to approach the students to case law applied to Spanish Civil Law; in this way, they will understand that the important thing is not to give the solution to case proposed but legal reasoning, through online discussions and in groups, getting a deeper knowledge of applicable rules and institutions.

Key words: case method, posthumous son, nasciturus, inheritance, Law Degree.

Recepción original: 22-7-2020

Aceptación original: 23-9-2020

\section{INTRODUCCIÓN}

Con la llegada de la pandemia causada por el coronavirus desde comienzos del año 2020 en España, en las Universidades españolas se ha visto la necesidad de modificar el actual sistema de enseñanza 
presencial hacia un modelo de docencia online o virtual ${ }^{1}$, que permita el distanciamiento social pero que, mediante el uso de herramientas digitales, los docentes puedan continuar con la enseñanza universitaria con normalidad y los alumnos adquieran los conocimientos y destrezas necesarios para la titulación que cursan, sin perder por ello calidad ni contenidos y/o competencias esenciales para su futuro profesional.

Concretando lo anterior, cabe recordar que durante décadas, el método tradicional de enseñanza del Derecho en las Universidades españolas y, en especial en los que respecta a la asignatura de Derecho Civil, era el de la exposición teórica de los contenidos a través de las clases magistrales. Pero desde hace una década y con los planes de estudio del Grado en Derecho surgidos en el Espacio Europeo de Educación Superior (EEES), se vio la necesidad de propiciar una metodología más activa y dinámica, por la que el alumnado universitario fuera el verdadero protagonista del proceso de enseñanzaaprendizaje; desarrollando en él competencias generales y específicas, muy novedosas hasta entonces respecto de los antiguos planes de la Licenciatura en Derecho.

Por dicho motivo, mediante un supuesto-tipo muy frecuente en materia de Derecho de Familia y Herencia desde Roma hasta nuestros días, vamos a demostrar cómo el término "competencia» aparece como un concepto propio que refleja tanto el papel fundamental de los estudiantes de Derecho en dicho proceso como la necesidad de que estos no sólo se formen en determinados conocimientos jurídicos, sino que ello lo completen con una serie de destrezas y habilidades sociales que la van a servir a la hora de desarrollar su profesión como futuros juristas, una vez que se enfrenten a las dificultades propias del mercado laboral actual.

En este punto, la actividad propuesta en el presente trabajo se enmarca dentro de la asignatura del Grado en Derecho denominada "Derecho Civil", la cual aborda distintas materias (entre otras abordadas en este supuesto, podemos señalar: matrimonio, filiación y herencia), y tiene por finalidad demostrar cómo los contenidos teóricos o conceptuales que se van desarrollando a lo largo del curso, parten de las soluciones dadas por la jurisprudencia actual a través del casuismo.

1 Sobre la enseñanza universitaria online a causa del COVID-19, vid. CABRERA, L. (2020), "Efectos del coronavirus en el sistema de enseñanza: aumenta la desigualdad de oportunidades educativas en España". Revista de Sociología de la Educación (RASE), vol. 13, n. ${ }^{\circ}$ 2, especial COVID-19: 114-139. En línea: https:// dialnet.unirioja.es/servlet/articulo? codigo $=7384620$ 
Por lo que es en este punto en el que entendemos que el método del caso, no sólo va a facilitar las discusiones grupales de forma presencial -cuando la situación actual de pandemia lo permita- y en el aula virtual de la materia sino que, a su vez, va a generar una serie de reglas generales muy útiles para comprender la asignatura, que parte de las reglas e instituciones aplicables a casos reales como el que citaremos y que ocurrieron desde Roma. De este modo, es nuestra intención que los discentes aprendan a razonar para que al acabar sus estudios universitarios, sean capaces de resolver supuestos reales.

Por tanto, esta actividad de Derecho Civil basada en la casuística romana que proponemos posee una doble finalidad: a) En primer lugar, que los alumnos comprendan la materia impartida en las clases online, especialmente, en lo que se refiere al matrimonio, al divorcio, a los hijos (nacidos, póstumos y/o nasciturus) y a la herencia; b) en segundo término, que los estudiantes sean capaces de razonar y aportar la solución más ajustada a Derecho, siempre teniendo muy presente las diversas variables que se suceden en este caso, esto es, la existencia de dos matrimonios consecutivos, los hijos nacidos y los que no, el haberse divorciado de la primera unión marital y el no haber otorgado testamento.

\section{OBJETIVOS PLANTEADOS Y CONTENIDOS PROPUESTOS EN EL SUPUESTO DE DOBLE MATRIMONIO, NASCITURUS E HIJO PÓSTUMO ANTE LA HERENCIA}

\section{II.A. Justificación}

Tal y como adelantábamos, con los nuevos planes de estudio se exige que los estudiantes de Derecho se formen en una serie de competencias y habilidades básicas, que le lleven a la consecución del Grado. Por dicho motivo, creemos imprescindible aportar unas breves referencias sobre el alcance de las citadas competencias ${ }^{2}$ para poder aplicarlas al Derecho Civil (ya sea Derecho de Familia o Herencia) y al Derecho Romano, pues lo anterior nos ayudará a comprender los objetivos y contenidos planteados con la puesta en práctica de esta actividad online sobre doble matrimonio romano, hijos, y ausencia de testamento.

En este ámbito, nos interesa tratar de definir la voz «competencia» ya que se trata de una palabra ambigua que ha venido planteando no pocas discusiones doctrinales en cuanto a su alcance, 
puesto que no resulta fácil acotar su concepto ${ }^{3}$. Una definición general nos la aporta Sobrado ${ }^{4}$ cuando, al tratar esta materia, sostiene textualmente que se considera competente quien tiene no sólo conocimientos, sino también aptitudes, intereses y destrezas para el ejercicio de un campo ocupacional por lo que posee capacidad para solucionar problemas y situaciones laborales de un modo autónomo y flexible; mientras que $\mathrm{Bretel}^{5}$, mantiene literalmente que una persona competente es aquella que posee las cualidades, los requisitos necesarios, la disposición y la creatividad para hacer algo cada vez mejor y para dar razón y justificar lo que hace, y de ahí la importancia del autoanálisis y la autoevaluación de las propias destrezas y habilidades.

Por tanto y, como ya tuvimos ocasión de demostrar ${ }^{6}$, el concepto de "competencias" aplicado al Título oficial de graduado en Derecho se relaciona con la necesidad de que los estudiantes adquieran unos conocimientos jurídicos básicos, que les sirvan para resolver desde un punto de vista legal cada caso concreto, junto a una serie de aptitudes y habilidades fundamentales para el desarrollo de su profesión en el mercado laboral, fomentándose cada vez más el uso de herramientas digitales pues los juzgados españoles han ido implementando en los últimos años los procedimientos judiciales online, el expediente judicial digital y el uso del buzón "LEXnet" para enviar notificaciones y recibirlas, de parte de todos los actores implicados (abogados, procuradores, médicos forenses, policía y el propio juzgado).

Veamos, pues, cuáles son esos contenidos jurídicos básicos y aptitudes necesarias en el caso que estamos analizando.

\section{II.B. Objetivos planteados para aplicar el método del caso}

Con la puesta en práctica de una metodología activa en la enseñanza virtual del Derecho Civil y su casuística jurisprudencial en materia de Derecho de Familia y Herencia, nuestro objetivo principal es adentrar al alumnado universitario en las reglas y princi-

3 NAVÍO, A. (2005), "Propuestas conceptuales en torno a la competencia profesional». Revista de Educación, 337: 213-234; TEJADA, J. (1999), "Acerca de las Competencias profesionales (I)». Revista Herramientas, 56: 20-21.

4 SOBRADO, L. (2002), "Acreditación de las cualificaciones profesionales», en Seminarios de la Sociedad Española de Pedagogía. En línea: http://www.redined.mec. es/oai/indexg.php?registro=014200430571

5 BRETEL, L. (2005), "Evaluación de Competencias». En línea: http:// breteleandocompeten.blogspot.com/2005/09/evaluacin-de-competencias.html

6 GONZÁlEZ LOSADA, S.; MUÑOZ CATALÁN, E., (2010), "Análisis de las competencias de los estudiantes de Derecho en los nuevos planes de estudio de Andalucía en el EEES», XXI. Revista de Educación, 12: 147-162. 
pios que tradicionalmente han venido siendo aplicados en el ordenamiento jurídico español, tras una interpretación del Código $\mathrm{Civil}^{7}$ y las reglas del Derecho Romano que le anteceden. Lo anterior servirá al discente para, en segundo momento del proceso online de enseñanza-aprendizaje y en aras a fomentar dichas competencias, tratar de razonar jurídicamente sobre el supuesto planteado, consiguiendo un conocimiento más profundo de las reglas e instituciones aplicables, y ofreciendo la solución más acorde a Derecho; siendo previa, la decisión de las acciones y recursos procesales que tutelan a las partes en el litigio.

En este punto, resulta interesante conocer el contenido íntegro del supuesto a resolver en la clase virtual, así como el de otro caso práctico de Derecho Romano similar que tuvo lugar en Roma, puesto que, de forma comparativa, entendemos que nos ayudarán a comprender mejor las reglas aplicables:

«Patricio, padre de tres hijos que tuvo en su primer matrimonio y casado en segundas nupcias con Claudia, fallece cuando su actual mujer está embarazada de cinco meses. Las dudas que nos plantea este caso son las siguientes: ¿cree Ud. que el hijo póstumo de Patricio y Claudia tendría algún derecho en la herencia de su padre, o solo correspondería a los tres hijos anteriores de Patricio? ¿qué tendría que ocurrir para que así fuera? ¿en qué momento se produciría la adquisición de derechos por el futuro hijo? (Hijo póstumo y herencia: Supuesto $n^{\circ}$ 6, Lasarte, C., 2010, p. 1578)».

«Un ciudadano romano, padre de familia, después de permanecer una larga temporada en España y de haber contraído matrimonio con una española, regresó a Roma dejando en España a su mujer que se encontraba encinta. Al llegar a Roma, contrajo de nuevo matrimonio con una romana sin preocuparse de notificar a la española que quería divorciarse de ella. De la unión con la mujer española nació un hijo y otro de la unión con la romana. Muerto el romano sin testar, los peritos en Derecho se plantean la cuestión de cuál de los dos matrimonios será válido y si debe heredar el hijo de la española o el hijo de la romana (Supuesto real del jurista: Cicerón, De oratore, $1.40 .183 ; 1.56 .238)^{9}{ }^{\natural}$.

Por lo anterior, podemos afirmar que lo que se pretende es que los estudiantes reflexionen grupalmente sobre las distintas soluciones propuestas, fomentando el trabajo colaborativo tanto en su ver-

7 BERCOVITZ RODRÍGUEZ-CANO, R. (Coord.) (2013). Comentarios al Código Civil. Navarra: Editorial Aranzadi.

8 LASARTE, C., (2010). Curso de Derecho Civil Patrimonial. Madrid: Editorial Tecnos, 157.

9 GARCÍA GARRIDO, M.J. (2019). Derecho Privado Romano -Casos-accionesinstituciones. Madrid: Editorial Sanz y Torres. 
sión presencial como en la online. Entendiendo, en todo caso, que lo importante no es encontrar la solución a los problemas matrimoniales, sucesorios y de herederos que el caso que analizamos plantea -ya que hay supuestos que admiten más de una solución justa-, pues lo que interesa es el razonamiento basado en argumentos jurídicos y criterios procesales que llevan al jurista a adoptar una determinada decisión aplicando las reglas del Derecho Civil.

Y, solo de este modo, consideramos que los alumnos se familiarizan con instituciones tradicionales tan importantes desde Roma como el matrimonio o la sucesión, y valorarán la importancia que tiene el ser llamado a la herencia en los distintos casos. Así como se reflexionará, de manera crítica por parte del profesorado, sobre otras estrategias de enseñanza diferentes a la tradicional o expositiva, especialmente desde que en marzo del año 2020 se produjo la suspensión de las clases en las Universidades españolas presenciales a causa de la enfermedad contagiosa del COVID- $19^{10}$ y la decisión de continuar con el curso a distancia y su evaluación online, mediante el manejo de herramientas de aprendizaje online.

\section{II.C. Contenidos conceptuales, procedimentales y actitudinales}

En este apartado, vamos a reparar en cuáles son los contenidos que nosotros creemos que se abordarán durante el desarrollo de esta actividad práctica y que ayudarán en el desarrollo de las referidas competencias; diferenciando entre los contenidos conceptuales («el saber»), procedimentales («saber hacer») y actitudinales («saber ser o comportarse»), los cuales, entendemos que resultan esenciales y se exponen resumidamente a través del siguiente esquema que ofrecemos con fines didácticos:

- Contenidos conceptuales:

- Características del matrimonio según nuestro Código Civil español.

- La figura de la separación o divorcio.

- Importancia de la herencia.

10 En cuanto a los problemas iniciales de la enseñanza online en las Universidades españolas presenciales motivada por la pandemia del coronavirus, vid. ROGERO-GARCÍA, J. (2020), "La ficción de educar a distancia". Revista de Sociología de la Educación (RASE), vol. 13, n. ${ }^{\circ}$ 2, especial COVID-19: 174-182. En línea: https://dialnet.unirioja.es/servlet/articulo?codigo=7384621 
- La figura del testamento y sus ventajas.

- La sucesión intestada o sin testamento.

- Las reservas hereditarias como institución jurídica: La reserva vidual.

- Consideración jurídica de los hijos según sean nacidos de un matrimonio u otro: especial referencia al nasciturus y postumus.

- Adquisición de derechos por el futuro hijo.

- El casuismo sobre Derecho de Familia y Herencia en la jurisprudencia actual.

- Las motivaciones y fundamentos de las decisiones jurisprudenciales.

- Legislación aplicable.

- Contenidos procedimentales:

- Análisis pormenorizado de los hechos.

- Determinación de los sujetos que intervienen en el caso: nacidos y no nacidos, póstumos y no póstumos.

- Estudio de la institución matrimonial, la separación y la herencia.

- Comprensión de las reglas, principios e instituciones jurídicas que son aplicables al caso propuesto.

- Exposición de las posibles acciones y medios de defensa de cada una de las partes para reclamar la herencia o tutelar al hijo no nacido.

- Análisis de las respuestas dadas por los tribunales de justicia sobre las distintas cuestiones planteadas.

- Elección razonada de la solución dada al caso, de entre las posibles, y su comparativa con supuestos-tipo ocurridos desde el Derecho Romano.

- Reflexión final sobre los contenidos abordados en el supuesto y sobre la estrategia didáctica empleada.

- Contenidos actitudinales:

- Concienciarse de la importancia que tiene para un jurista el razonar, aportando argumentos fundados en Derecho Civil. 
- Interés por conocer de qué manera se resolvían los supuestos en el Imperio romano, para tratar de aplicar las reglas a casos actuales.

- Predisposición a la consulta de los textos jurídicos y a su utilización.

- Participación con interés y agrado en los trabajos en común y en los debates que se realicen en el aula, tanto presencialmente como online, en la plataforma virtual de la materia (espacio habilitado para subir los trabajados, foros, etc.).

- Valoración positiva de las soluciones aportadas en clase por los distintos compañeros, aplicando siempre las alternativas que ofrece nuestro Código Civil.

- Reflexión y valoración de cómo, la regulación del matrimonio y la herencia en nuestros días, es fruto de la lucha por solucionar los problemas a lo largo de la Historia.

\section{DESARROLLO DE LA ACTIVIDAD EN CLASE, DE FORMA PRESENCIAL Y VIRTUAL}

\section{III.A. Primera sesión}

Como posteriormente comentaremos, los alumnos siguen un sistema de evaluación continua (60\% examen final $+40 \%$ evaluación continua). Y que, debido a la pandemia y a la potenciación de las clases online, la misma será mayoritariamente de manera virtual (mediante la herramienta Zoom o Google meet, entre otras) y haciendo usos de las herramientas digitales (foros, chat, etc. de aulas virtuales, como por ejemplo, Moodle).

En cuanto a la evaluación continua, la misma consiste en dar solución a un caso práctico planteado. Teniendo presente que para su desarrollo vamos a emplear un total de ocho horas (cuatro presenciales y otras cuatro online, o si la situación de pandemia no lo permite, todas se impartirán a distancia), se da comienzo a la primera sesión de presentación del caso práctico cuya duración es de dos horas, exponiendo sucintamente el contenido teórico analizado en clases anteriores y que va a servir de base para la resolución del supuesto propuesto sobre reparto hereditario cuando hay hijos de varios matrimonios y uno de ellos tiene la consideración de concebido y no nacido, así como póstumo. En este punto, cabe precisar que la evaluación se hará al final de la segunda sesión programada, te- 
niendo en cuenta: el trabajo individual inicial y posteriormente en grupo, los debates online, el manejo de las herramientas, el cumplimiento de los plazos oficiales, etc.

En este contexto, se hará uso del ordenador y se proyectará en el aula virtual una presentación power-point sobre matrimonio y herencia que servirá de guion, así como el docente irá preguntando a los estudiantes sobre otras características que podrían añadir a cada institución estudiada, que comenten casos reales, noticias relacionadas, etc.

A continuación, se entregará al alumnado -por escrito o en el apartado de recursos del aula- el supuesto práctico para que lo realicen. La actividad, en un primer momento, se desarrollará individualmente y consistirá en la elaboración de un esquema-resumen de las relaciones de parentesco que se suceden, así como del problema jurídico planteado sobre el matrimonio válido, hijos legítimos y el reparto de la herencia entre los descendientes, que se entregará al finalizar la sesión. Es aquí donde nos interesa examinar el texto jurídico tradicional al que aludimos, y del que hemos modificado solo los nombres de los intervinientes, y destacar las palabras que entendemos que nos van a dar las claves para resolver el caso:

«Patricio, padre de tres hijos que tuvo en su primer matrimonio y casado en segundas nupcias con Claudia, fallece cuando su actual mujer está embarazada de cinco meses. Las dudas que nos plantea este caso son las siguientes: ¿cree Ud. que el hijo póstumo de Patricio y Claudia tendría algún derecho en la herencia de su padre, o solo correspondería a los tres hijos anteriores de Patricio? ¿qué tendría que ocurrir para que así fuera? ¿en qué momento se produciría la adquisición de derechos por el futuro hijo? (Hijo póstumo y herencia: Supuesto $\mathrm{n}^{\circ} 6$, Lasarte, C. p. 157)».

Para responder a dichas cuestiones planteadas, creemos preciso tener presente los artículos del Código Civil español que regulan esta materia, y que exponemos seguidamente ${ }^{11}$ :

Artículo 959: "Cuando la viuda crea haber quedado encinta, deberá ponerlo en conocimiento de los que tengan a la herencia un derecho de tal naturaleza que deba desaparecer o disminuir por el nacimiento del póstumo".

Artículo 964: "La viuda que quede encinta, aun cuando sea rica, deberá ser alimentada de los bienes hereditarios, habida consideración a la parte que en ellos pueda tener el póstumo, si naciere y fuere viable".

11 VLEX, "De las precauciones que deben adoptarse cuando la viuda queda encinta" [En línea]: https://libros-revistas-derecho.vlex.es/vid/articulo-963-231026 
De las Clases magistrales al MÉtodo Del CASO ANTE SUPUESTOS...

Artículo 29: "El nacimiento determina la personalidad; pero el concebido se tiene por nacido para todos los efectos que le sean favorables, siempre que nazca con las condiciones que expresa el artículo siguiente".

Artículo 811: "El ascendiente que heredare de su descendiente bienes que éste hubiese adquirido por título lucrativo de otro ascendiente, o de un hermano, se halla obligado a reservar los que hubiere adquirido por ministerio de la ley en favor de los parientes que estén dentro del tercer grado y pertenezcan a la linea de donde los bienes proceden".

De la conjugación de dichos preceptos, se extraen las siguientes premisas aplicables a nuestro caso: $1^{\circ}$ La viuda que quede encinta deberá ser alimentada de los bienes hereditarios, habida consideración a la parte que en ellos pueda tener el póstumo, si naciere y fuere viable. $2^{\circ} \mathrm{El}$ concebido se tiene por nacido para todos los efectos que le sean favorables, si nace conforme a lo dispuesto en el artículo 30 Código Civil: que sea un nacimiento con vida y que tenga lugar el entero desprendimiento del seno materno. (y, por tanto, tiene capacidad jurídica, personalidad, y "es persona"). $3^{\circ}$ Importancia del testamento y las reservas hereditarias, para no lesionar el derecho al reparto hereditario cuando hay varios matrimonios e hijos de distinta unión.

En este ámbito, se entiende por "reserva hereditaria" ${ }^{12}$ la limitación de origen a la libertad del testador, que determina el destino que han de seguir determinados bienes que integran el caudal relicto, asegurando que sean adquiridos por miembros de la familia de la que proceden. En cuanto a su regulación jurídica, debemos acudir a lo dispuesto en el artículo 811 y 968-980 del Código Civil español donde se diferencia entre reserva lineal y vidual.

Sobre esta última, los estudiantes deben conocer que es la más usual en este tipo de casos, y que el causante debió haberla realizado en el supuesto práctico que nos ocupa. Por tanto, además de la reserva impuesta en el artículo 811 del Código Civil, hay que tener presente que el viudo o viuda que pase a segundo matrimonio estará obligado a reservar a los hijos y descendientes del primero la propiedad de todos los bienes que haya adquirido de su difunto consorte por testamento, por sucesión intestada, donación u otro cualquier título lucrativo; pero no su mitad de gananciales. Y, en lo que respecta a su fundamento jurídico, resulta muy útil la Sentencia del Tribunal

12 COLEX. IBERLEY (2016), "Regulación de las reservas hereditarias como institución fundamental de la sucesión forzosa en el Código Civil” [En línea]: https://www.iberley.es/temas/regulacion-reservas-hereditarias-codigo-civil-59790

() UNED. Revista de Derecho UNED, núm. 27, 2021 
Supremo de 22 de junio de 1995, número 609, Sección $1^{a}$. Recurso número 1440/1992 sobre renuncia a la reserva viudal por hijos mayores de edad del primer matrimonio, pues como nos advirtiera el Tribunal Supremo hace ya varias décadas:

"La razón de ser de esta institución, con amplios antecedentes en el Derecho romano y en el Derecho histórico español, (Fuero Juzgo, Fuero Real, Partidas y Leyes de Toro) podemos concretarla como una limitación a la facultad de disponer impuesta al cónyuge bínubo, con la finalidad de proteger los intereses de los hijos y descendientes del primer matrimonio, en relación con los bienes procedentes gratuitamente de su progenitor fallecido, frente a la posible presencia de otros hijos nacidos de la segundas nupcias».

De manera comparativa y con la finalidad última de que los estudiantes comprendan mejor las reglas e instituciones aplicables actualmente al caso, nos interesa brevemente mostrarles cuál es el contenido básico de la otra práctica similar sobre Derecho de Sucesiones y Matrimonio en Roma que más arriba hemos indicado.

Teniendo en cuenta su contenido y, tras varias lecturas comprensivas (dado que se trata de la traducción de un texto jurídico del latín al español), cabe distinguir entre los siguientes hechos ${ }^{13}$ : a) El primero, que la herencia correspondería al hijo legítimo del matrimonio con la española al haber nacido de un matrimonio legal; b) el segundo, que según las reglas del Derecho Civil y por lo que se refiere a las relaciones de parentesco, los hijos heredarían con prioridad respecto a las mujeres y ninguna de las dos madres serían sucesoras; c) sin embargo, en cuanto al segundo hijo, sólo heredaría si el romano hubiera notificado a la primera mujer el repudio; si bien hemos visto, que dicho dato no aparece en el caso, por lo que podemos considerar al segundo como ilegítimo y no heredero.

Dicha sesión concluirá abriéndose un turno de debate en la clase online, en el que se tratarán las primeras impresiones de los discentes. El profesorado planteará una serie de cuestiones que deberán traer trabajadas para la próxima clase, así como se informará al alumnado de que dispone de diez días para participar en el foro-debate: "Patricio y el reparto de su herencia: nasciturus y postumus», que se plantea en la plataforma virtual Moodle de la asignatura, así como que cada estudiante debe subir el archivo de su trabajo al espacio individual habilitado en el aula online de la materia; insistién-

13 MUÑOZ CATALÁN, E. (2013), “EEES, competencias y aprendizaje del Derecho Romano mediante el método del caso (I): El doble matrimonio". REJIE: Revista Jurídica de Investigación e Innovación Educativa, 7: 51-62 [En línea]: http:// www.eumed.net/rev/rejie 
doles en la importancia de que formen grupos de cinco alumnos para la próxima sesión, y de su participación activa e interés mostrado, de cara a la calificación final de esta práctica.

\section{III.B. Segunda sesión}

El docente empezará la segunda sesión de otras dos horas, resumiendo lo explicado en la clase anterior y recordando las preguntas planteadas previamente, en concreto, en lo que concierne a los siguientes puntos a abordar:

- ¿Cree Ud. que el hijo póstumo de Patricio y Claudia tendría algún derecho en la herencia de su padre, o solo correspondería a los tres hijos anteriores de Patricio?

- ¿Qué tendría que ocurrir para que así fuera?

- ¿En qué momento se produciría la adquisición de derechos por el futuro hijo?

Igualmente, tras organizarse en grupos de cinco alumnos, cada uno de sus miembros compararán las respuestas y así se unificarán los criterios. Posteriormente, cada equipo expondrá sus conclusiones y se realizará una puesta en común, mediante la herramienta de docencia online. Al final de esta actividad, por parte del profesorado se aportará un esquema-resumen de las instituciones abordas, que igualmente dejará en formato digital en el aula, y se ofrecerá la solución que se considera más ajustada a Derecho, razonando con los estudiantes sobre otros posibles enfoques.

En cuanto a lo primero, cabe señalar de manera sucinta las diferentes variables que sirven para resolverlo aplicando nuestro vigente Código Civil español:

- Primer matrimonio y descendientes (legítimos).

- Disolución del matrimonio inicial.

- Segundo matrimonio y descendiente, nasciturus y postumus (protección hasta que nazca y adquiera plena capacidad para aceptar la herencia. Legítimo).

- Fallece el padre (causante y no testador): Sin hacer testamento y sin realizar reservas hereditarias.

- Reparto de la herencia entre todos los hijos y la segunda mujer. 
Finalmente, en cuanto al caso jurisprudencial romano, podemos advertir las siguientes circunstancias que entendemos nos ayudan a resolverlo ya con más detalle en esta segunda sesión programada: a) El hijo del primer matrimonio se consideraría como legítimo, al haber nacido de un matrimonio romano justo y legalmente conformado, sin embargo, el segundo no se sabe si es o no legítimo pues para ello haría falta que el fallecido hubiese enviado a la española el libelo de «repudio»; b) en cuanto a las relaciones de parentesco y, según las reglas del Derecho Civil y de la bonorum possessio, los hijos heredarían con prioridad respecto a las mujeres; por lo que ninguna de las dos heredarían; c) en definitiva, la herencia correspondería al hijo legítimo del matrimonio con la española pero, en cuanto al segundo hijo, sólo sucedería si el romano hubiera notificado a la primera mujer el repudio; hecho que en el caso no aparece, por lo que podemos considerarlo como ilegítimo y no heredero.

Para acabar, se expondrá en el foro virtual el resultado de la experiencia propuesta y, finalmente, se darán unas conclusiones finales y se realizarán propuestas de mejora. Y, todo ello, quedará grabado y colgado en Moodle para aquellos alumnos que quieran volver a visualizarlo o si no pudieron asistir, tengan el mecanismo para seguir la materia sin problema.

\section{TEMPORALIZACIÓN RECOMENDADA}

En resumen, de lo anterior se extrae que para la puesta en práctica de esta actividad en la que aplicamos el método del caso al Derecho Civil matrimonial y hereditario, se requerirán dos sesiones de dos horas cada una -siendo igual para docencia presencial que digital-, empleando un total de cuatro horas presenciales y otras cuatro horas online:

- La primera sesión consistirá en un breve resumen de las instituciones abordadas en el supuesto planteado (causante, sucesión sin testamento, matrimonios, separación, herencia, reservas hereditarias, nasciturus, postumus e hijos de varios matrimonios, etc.); lectura comprensiva del caso propuesto; $\mathrm{y}$ esquema-resumen de las relaciones de parentesco tratadas en el caso práctico y primeras impresiones de los estudiantes.

- La segunda sesión, comenzará con un recordatorio de lo visto en la clase anterior para, a continuación, trabajar en equipos comparando cada uno de sus miembros las respuestas que han dado (tanto por escrito presencialmente como en la pla- 
taforma online), para tratar de unificar criterios (si heredan todos los hijos y condiciones en ese caso; si hereda la segunda mujer o la primera; qué ocurre si no hay testamento; etc.). Finalmente, se hará una puesta en común por grupos, se ofrecerán unas conclusiones finales y unas propuestas de mejora.

Al mismo tiempo y, haciendo uso de las TIC's, se planteará un foro de debate virtual sobre la solución más ajustada a Derecho en el caso concreto; los estudiantes accederán a él las veces que estimen conveniente y participarán activamente en el foro habilitado durante diez días. Tras ello, el docente deberá tener en cuenta la participación e interés del alumnado por resolver el supuesto valorando, en cualquier caso, las competencias adquiridas mediante la puesta en práctica de este tipo de actividades y cuya función esencial fueron expuestas al comienzo de nuestro trabajo.

\section{RECURSOS}

En este punto, nos interesa reparar en los recursos que nosotros personalmente entendemos que ayudarán al buen desarrollo de esta práctica civil, diferenciando entre recursos materiales, ambientales, estructurales y los propios del ámbito universitario:

\section{V.A. Recursos materiales (material «aprender a aprender»)}

- Hijo póstumo y herencia: Supuesto $\mathrm{n}^{\circ}$ 6, Lasarte, C., 2010, p. 157.

- Supuesto real del jurista: Cicerón, De oratore, 1.40.183; 1.56.238.

- Esquema-resumen de las relaciones de parentesco que se enumeran en el caso.

- Libros de consulta.

- Textos legales.

- Jurisprudencia:

- Sentencia del Tribunal Supremo de 22 de junio de 1995, número 609, Sección $1^{\text {a }}$. Recurso número 1440/1992. Renuncia a la reserva viudal por hijos mayores de edad del primer matrimonio. 
- Sentencia del Tribunal Constitucional 53/1985, de 11 de abril. BOE núm. 119, de 18 de mayo de 1985. Sobre el derecho a la vida y nasciturus.

- Ordenador, proyector, y pizarra (en caso de docencia presencial).

- Plataforma virtual Moodle y recursos digitales a su disposición: foro, chats, etc. (en caso de docencia online, que será lo más frecuente por la situación de pandemia actual).

Tabla n. 1. Recursos para la propuesta metodológica activa diseñada

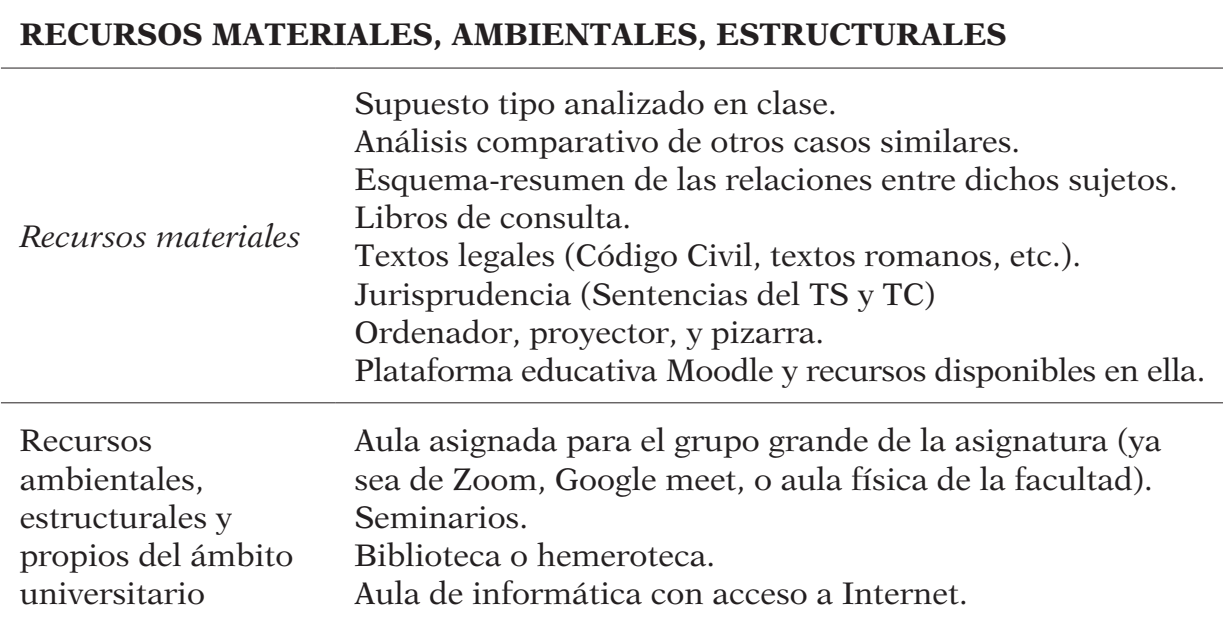

Fuente: Elaboración propia

\section{V.B. Recursos ambientales, estructurales y propios del ámbito universitario}

Los espacios a utilizar serán, además del aula virtual de la asignatura -en caso de enseñanza virtual a causa del coronavirus-, el aula asignada para el grupo grande de la asignatura a lo largo del curso y los seminarios de la Facultad de Derecho reservados para los días de actividades complementarias, prácticas o tareas en pequeños grupos -si continúan las clases presenciales-. Del mismo modo que se podrá utilizar la biblioteca o hemeroteca general, tanto presencial como a distancia, y, siempre que sea conveniente, se utilizará el aula de informática de la Universidad con acceso a Internet. 


\section{EVALUACIÓN: CRITERIOS E INSTRUMENTOS}

Los alumnos siguen un sistema de evaluación continua (60\% examen final+40\% evaluación continua). El mismo, no varía siendo una enseñanza online, todo lo contrario, pues desde la pandemia se fomenta aún más en todas las Universidades españolas este tipo de evaluación.

Como avanzábamos, la evaluación continua de las actividades propuestas se hará al final de la segunda sesión del caso práctico programada, teniendo en cuenta: el trabajo individual inicial y posteriormente en grupo, los debates, el manejo de las herramientas (como el foro), el cumplimiento de los plazos oficiales, etc.. Por ello, nosotros hemos definido los siguientes criterios de valoración de los estudiantes:

- Domina y define los conceptos básicos en materia matrimonial y hereditaria, tanto como se configura en el Código Civil actual como en el Derecho Romano.

- Sabe cuál es la relación de parentesco, a la hora de sucederse en la herencia aun sin testamento.

- Conoce las distintas modalidades de matrimonio desde Roma.

- Diferencia entre separación y divorcio, hijo nacido, póstumo o nasciturus.

- Maneja las claves de la reserva hereditaria.

- Emplea las fuentes básicas de información del Derecho Civil.

- Identifica, en situaciones tipo, las variables que determinan la aplicación de unos principios u otros.

- Busca, maneja y aplica, al caso concreto, la normativa legal que resulta aplicable actualmente.

- Demuestra el dominio de las técnicas de resolución de supuestos.

- Posee una actitud activa en clase y participa en las diferentes actividades (presenciales y virtuales), demostrando esfuerzo, constancia y tesón. 


\section{CONCLUSIONES Y RESULTADOS}

A modo de conclusión, finalizamos la presente propuesta de metodología activa online aplicada a la enseñanza del Derecho de Familia y Sucesiones, resaltando la necesidad actual de una docencia universitaria virtual a causa de la pandemia mundial que estamos sufriendo a nivel mundial desde comienzos del año 2020 por la enfermedad contagiosa del coronavirus. En este sentido, hemos llegado a los siguientes resultados:

$1^{\circ}$ Con el trabajo realizado, consideramos que se ha demostrado la importancia que hoy tiene aplicar este tipo de metodologías online -basadas en el método del caso- al Derecho Civil frente a las tradicionales clases magistrales, pues de este modo los estudiantes que comienzan sus estudios de Grado en Derecho, conocerán mejor el Derecho matrimonial y sucesorio desde su regulación en el Derecho Romano hasta su proyección actual; Así como desarrollarán una serie de competencias que hoy resultan esenciales para razonar y aplicar las reglas del Código Civil español a supuestos como el que hemos propuesto durante el diseño de dicha práctica.

$2^{\circ}$ El caso planteado, tal y como hemos detallado, parte de la importancia de proteger jurídicamente a los hijos habidos en distintos matrimonios, e incluso aquellos nasciturus y postumus a la muerte del padre. De ahí que, en aras a responder legalmente al supuesto analizado, se puedan destacar las diferentes variables que sirven para resolverlo aplicando nuestro vigente Código Civil español, de entre las que señalamos: a) La existencia de un primer matrimonio y descendientes (legítimos); b) la disolución del matrimonio inicial; c) el segundo matrimonio y la consideración de su descendiente como nasciturus y postumus (protección hasta que nazca y adquiera plena capacidad para aceptar la herencia: hijo legítimo); d) el fallecimiento del causante y no testador, padre de los mismos, sin otorgar testamento y sin realizar reservas hereditarias; e) así como las dificultades propias del reparto de la herencia entre todos los hijos, de uno y otro matrimonio, y también respecto de la segunda mujer y no la primera.

$3^{\circ}$ En tercer lugar, hemos determinado que precisamente con la implementación de este caso que aborda instituciones tan importantes hoy como el matrimonio, la separación o divorcio, la 
sucesión sin testamento, los hijos legítimos, la figura del póstumo y el nasciturus, demostramos cómo con los planes de estudio del Grado en Derecho y con las exigencias metodológicas surgidas desde el EEES y el cada vez más frecuente uso de herramientas digitales en la docencia universitaria española, se deben impulsar en el aula de Derecho Civil y las materias que lo integran (en especial, en lo que concierne al Derecho de Familia, Matrimonio y la Herencia) -tanto de forma presencial como mediante el manejo de la plataforma virtual-, una serie de competencias básicas en el alumnado de Derecho, las cuales, resultaban impensables hasta hace relativamente poco tiempo y que favorecen el aprendizaje, la oratoria, el razonamiento jurídico y la innovación.

$4^{\circ}$ En suma, concluimos admitiendo que mediante el aprendizaje online basado en casos -del tipo propuesto y su comparativa con otros tradicionales-, entendemos que se potencia un nuevo modo de transmitir los conocimientos jurídicos por parte del docente universitario, por el que no sólo se fomentan los contenidos conceptuales y procedimentales -importantes para el Derecho Civil-, sino también los actitudinales mediante el manejo de herramientas sociales, como pueden ser los foros, chats o las redes sociales. Y, solo de este modo, logramos de manera más práctica implicar al alumnado que estudia Derecho y favorecemos su reflexión y actitud crítica, especialmente en cuestiones sobre el Derecho de Familia y la Herencia.

Lo que consideramos que, muy especialmente, les servirá para cuando finalicen sus estudios jurídicos y se enfrenten a las duras condiciones económicas del actual mercado de trabajo a causa, especialmente, de la pandemia provocada por el COVID-19 y del futuro incierto que nos espera en España para los próximos meses y años en todas las esferas sociales.

\section{BIBLIOGRAFÍA}

\section{VIII.A. Referencias bibliográficas}

Bercovitz Rodríguez-Cano, R. (Coord.) (2013). Comentarios al Código Civil. Navarra: Editorial Aranzadi. 
Bretel, L. (2005), «Evaluación de Competencias». [En línea] http:// breteleandocompeten.blogspot.com/2005/09/evaluacin-de-competencias.html

Cabrera, L. (2020), "Efectos del coronavirus en el sistema de enseñanza: aumenta la desigualdad de oportunidades educativas en España”. Revista de Sociología de la Educación (RASE), vol. 13, n. ${ }^{\circ}$ 2, especial COVID-19: 114-139. En línea: https://dialnet.unirioja.es/ servlet/articulo?codigo $=7384620$

Domingo, R. (2002). Textos de Derecho Romano. Navarra: Thomson Aranzadi.

FERnández BujÁn, F. y García Garrido, M.J. (2019). Fundamentos Clásicos de la Democracia y la Administración. Madrid: Editorial Universitas.

García Garrido, M.J. (2019). Derecho Privado Romano -Casos-acciones-instituciones. Madrid: Editorial Sanz y Torres.

González Losada, S.; MuÑoz CataláN, E., (2010), «Análisis de las competencias de los estudiantes de Derecho en los nuevos planes de estudio de Andalucía en el EEES», XXI. Revista de educación, 12: 147-162.

Lasarte, C. (2010). Curso de Derecho Civil Patrimonial. Madrid: Editorial Tecnos.

Muñoz Catalán, E. (2013), “EEES, competencias y aprendizaje del Derecho Romano mediante el método del caso (I): El doble matrimonio". REJIE: Revista Jurídica de Investigación e Innovación Educativa, 7: 51-62 [En línea]: http://www.eumed.net/rev/rejie

NAvío, A. (2005), «Propuestas conceptuales en torno a la competencia profesional». Revista de Educación, 337: 213-234.

Rogero-García, J. (2020), "La ficción de educar a distancia". Revista de Sociología de la Educación (RASE), vol. 13, n. ${ }^{\circ}$ 2, especial COVID-19: 174-182. En línea: https:/dialnet.unirioja.es/servlet/ articulo?codigo $=7384621$

SoBRADO, L. (2002), «Acreditación de las cualificaciones profesionales», en Seminarios de la Sociedad Española de Pedagogía. [En línea] http://www.redined.mec.es/oai/indexg.php?registro=014200430571

TeJadA, J. (1999), «Acerca de las Competencias profesionales (I)». Revista Herramientas, 56: 20-30.

- (1999), «Acerca de las Competencias profesionales (II)» Revista Herramientas, 57: 8-14. 


\section{VIII.B. Fuentes jurisprudenciales, legislativas y/o electrónicas [Recuperadas: Octubre, 2020]}

Código Civil español (Edición vigente hasta el 22 de Julio de 2014) [En línea]: http://noticias.juridicas.com/base_datos/Privado/cc.html

COLEX. IBERLEy (2016), "Regulación de las reservas hereditarias como institución fundamental de la sucesión forzosa en el Código Civil" [En línea]: https://www.iberley.es/temas/regulacion-reservas-hereditarias-codigo-civil-59790

Constitución española de 1978 [En línea]: https://www.boe.es/buscar/ act.php?id=BOE-A-1978-31229

Ley 30/1981, 7 julio, por la que se modifica la regulación del matrimonio en el Código Civil y se determina el procedimiento a seguir en las causas de nulidad, separación y divorcio [En línea]: https:// www.boe.es/buscar/doc.php?id=BOE-A-1981-16216

Ley 13/2005, de 1 de julio, por la que se modifica el Código Civil en materia de derecho a contraer matrimonio [En línea]: https:// www.boe.es/eli/es/1/2005/07/01/13

Sentencia del Tribunal Supremo de 22 de junio de 1995, número 609, Sección $1^{\text {a }}$. Recurso número 1440/1992. Renuncia a la reserva viudal por hijos mayores de edad del primer matrimonio.

Sentencia del Tribunal Constitucional 53/1985, de 11 de abril (BOE núm. 119, de 18 de mayo de 1985). Sobre el derecho a la vida y nasciturus. [En línea] https://hj.tribunalconstitucional.es/es-ES/ Resolucion/Show/433

Textos jurídicos de Derecho Romano [En línea]: http://bib.us.es/derecho/recursos/pixelegis/areas/derechoRomano-ides-idweb.html

VLEX, "De las precauciones que deben adoptarse cuando la viuda queda encinta" [En línea]: https://libros-revistas-derecho.vlex.es/ vid/articulo-963-231026 
